\title{
ACTO DE PRESENTACIÓN DEL LIBRO HOMENAJE AL PROFESOR ANTONIO TORRES DEL MORAL
}

El 17 de enero de 2013 se presentó, en el Centro de Estudios Políticos y Constitucionales, el Libro Homenaje al profesor Antonio Torres del Moral, titulado Constitución y Democracia: ayer y hoy. El libro se compone de tres volúmenes, con 3.800 páginas, en las que han participado más de 180 académicos, españoles y extranjeros. La mayoría de ellos son constitucionalistas, aunque también hay muchos otros de áreas afines o que, simplemente, han participado en el libro por su relación con el profesor Torres.

$\mathrm{Al}$ acto de presentación asistieron, asimimo, un buen número de los autores partipantes, así como numerosas personas del ámbito académico, jurídico e intelectual, que desbordaron las salas del Centro que nos acogió.

Como pequeña contribución de esta Revista, que tantos años lleva dirigiendo Antonio Torres del Moral, hemos querido recoger aquí alguna de las intervenciones que tuvieron lugar en dicho acto, incluyendo la del propio homenajeado.

Carlos Vidal Prado Secretario de la Revista. 


\title{
INTERVENCIÓN DE YOLANDA GÓMEZ SÁNCHEZ
}

\author{
(Catedrática de Derecho Constitucional de la UNED)
}

Señor Director del Centro de Estudios Políticos y Constitucionales, Señor Rector Mgfco. de la UNED, autoridades, Profesor Torres del Moral, amigas, amigos.

Me corresponde iniciar a mí este turno de intervenciones en este entrañable acto de presentación del libro Constitución y Democracia: ayer y boy en homenaje al Profesor Torres del Moral, en un marco inigualable para cualquier constitucionalista, el Centro de Estudios Políticos y Constitucionales, que hoy nos acoge y donde además somos siempre recibidos con tanto afecto. Muchas gracias al Director de este Centro de Estudios por su hospitalidad.

Vengo yo a hablarles a todos ustedes fundamentalmente del Libro Homenaje que hoy presentamos, porque quienes me han de suceder en el uso de la palabra, harán una semblanza académica y personal del Profesor Torres del Moral, sin duda alguna, con más brillantez y acierto de la que yo pudiera hacer gala en estos minutos. Pero como verán ustedes enseguida, no es fácil, quizá ni siquiera es deseable, presentar un Libro Homenaje sin mencionar algunos de los valores de quién se ha hecho acreedor al mismo. Y en este sentido, diré que estamos hoy aquí para presentar una obra Homenaje, a un Profesor, a un Profesor que ha dedicado sin solución de continuidad casi cincuenta años de su vida a la Universidad española. Más de treinta de estos años, han sido prestando servicios en la UNED y, prácticamente todos ellos, como Director del hoy denominado Departamento de Derecho Constitucional, que actualmente tengo el honor de dirigir.

Creo que es este cargo que ahora ejerzo el que justifica que hoy esté yo aquí hablándoles a todos ustedes y no otros compañeros del Departamento de Derecho Constitucional y, también, de otros Departamentos, como el de Derecho Político, por ejemplo, con el que compartimos área, que reunirían, con toda seguridad, iguales o más méritos que yo misma, si es que yo tuviera alguno. Quizá puedo alegar en mi favor, la dirección del Profesor Torres del Moral de mi Tesis Doctoral cuando en 1982 me incorporé a la UNED, después de haber 
sido alumna suya en la Universidad Complutense. Creo que fue esa la primera Tesis Doctoral que dirigió, después de incorporarse a la UNED en 1981. Tesis a la que han seguido muchas más, un número relevante de las cuales, además, también han recibido el Premio Extraordinario de Doctorado.

Les decía hace un momento que este Libro Homenaje lo es a un Profesor. Un Profesor que ha dedicado su vida a la enseñanza y a la investigación. Puedo decirles a todos ustedes que, al menos en los últimos treinta años, que son en los que yo he coincidido y compartido con el Profesor Torres del Moral labores universitarias, no ha dejado nunca de estudiar, no ha dejado nunca de enseñar, y no ha dejado nunca de investigar. Ha sido, pues, la suya, una opción vital sin fisura alguna. Quiero pensar además que es una manera de vivir la Universidad intensa, continua, inasequible al desaliento, en un ámbito que se nos antojaba convulso años atrás. ¡No sabíamos lo que nos esperaba en 2011, ni en 2012! ¡Ni lo que vendrá en 2013! Esta manera de vivir la Universidad, digo, tiene mucho de lo que el Profesor Torres del Moral aprendió de su padre, maestro, en una no muy grande localidad de Andalucía.

Recuerdo que, hace ya más años de los que quisiera, nos contaba a algunos de nosotros que su padre había sido su maestro, su maestro en la Escuela; seguramente lo que el Profesor Torres del Moral aprendió en aquellos años marcó su deseo de enseñar y la forma en la que lo ha hecho a lo largo de su vida, aunando lo mejor del maestro y lo mejor del profesor universitario.

Les decía también, que el Profesor Torres del Moral ha sido Director del Departamento durante casi treinta años. Durante este tiempo, los logros del Departamento de Derecho Constitucional son incuestionables. Logros que esgrimo continuamente ante el Rector y el Vicerrector que corresponda por razón de la materia en el desenvolvimiento de mis funciones como Directora del Departamento, pero que sé, en justicia, que corresponden a una dirección previa a la mía, la del Profesor Torres del Moral.

Las Tesis Doctorales a las que he aludido, o la promoción del profesorado durante estas últimas décadas, son muestra evidente de estos logros. Pero con todo, es su labor como Director de la Revista de Derecho Político desde 1984, uno de los frutos de más calado y que tendrá mayor perdurabilidad. Cuando los números de la Revista de Derecho Político, en papel, se consideren y denominen por todos información predigital, y cuando otros números on-line les hayan sucedido, el nombre de su director, de Antonio Torres del Moral, seguirá unido a esta publicación, donde han encontrado un ámbito de libertad científica cientos de investigadores de múltiples nacionalidades a lo largo de casi tres décadas.

Debo ya encaminar mi intervención hacia el final de la misma. Ahora sí les contaré algo sobre el Libro Homenaje. A la idea inicial del Profesor Pedro Julio 
Tenorio de hacer un Libro Homenaje al Profesor Torres del Moral nos unimos, en seguida, los demás profesores del Departamento de Derecho Constitucional, aunque, es de justicia señalar, que la carga de trabajo en las múltiples labores ejecutivas ha sido realizada por los Profesores del Departamento Pedro Julio Tenorio Sánchez, Carlos Vidal Prado, Aurora Gutiérrez Nogueroles, con la inestimable ayuda de Leyre Burguera, Joaquín Sarrión y Hector Álvarez, y por nuestros queridos colegas el Profesor Luis Jimena de la Universidad de Valencia y el Profesor Javier Tajadura de la Universidad del País Vasco.

La convocatoria para esta obra Homenaje tuvo una respuesta amplísima; respondieron y enviaron sus aportaciones más de ciento setenta autores españoles y extranjeros. Los trabajos se han recogido a lo largo de 3.800 páginas organizadas en tres volúmenes y precedidas por sendos escritos del Rector Mgfco. de la UNED, Profesor Juan Gimeno Ullastres y del Director del Centro de Estudios Políticos y Constitucionales, Benigno Pendás, a los que agradecemos con toda sinceridad su inestimable colaboración.

A lo largo de las 3.800 páginas se recogen trabajos de muy diferente ámbito, pero todos ellos, como la propia obra del Profesor Torres del Moral, son perfectamente ubicables en el título que los cobija Constitución y Democracia: ayer y hoy, y diría yo más: Constitución y Democracia por siempre.

Muchas gracias a todos los que han encontrado tiempo para enviarnos su trabajo; muchas gracias también a todos aquellos que por distintas circunstancias no han podido hacerlo, pero nos han enviado su afecto y su adhesión al proyecto; muchas gracias a todos ustedes, que también han encontrado un espacio en sus quehaceres para acompañarnos esta tarde.

Y por último, y sabiendo del apego del Profesor Torres del Moral por la poesía, donde también ha demostrado maestría, y no teniendo yo, por el contrario, ni destrezas ni habilidades en tal campo, he pedido prestados unos versos para finalizar mi intervención. Dicen:

En el Maestro la experiencia

Es como el sol que se pone

Aunque el ocaso aparenta

Va con su luz a otros hombres.

Muchas gracias al Profesor Torres del Moral por una vida dedicada a la Universidad que, en tantos de nosotros, ha dejado su huella.

Muchas gracias. 


\section{SEMBLANZA PERSONAL Y PROFESIONAL DEL PROFESOR TORRES DEL MORAL}

(Intervención de Pedro Tenorio. Catedrático de Derecho constitucional. UNED)

Sr. Director del Centro de Estudios Políticos y Constitucionales, don Benigno Pendás, Sr. Rector Magnífico de la UNED, don Juan Gimeno Ullastres, Excmo. Sr. Don Miguel Herrero de Miñón, Ponente de la Constitución, y demás miembros de la mesa, Excelentísimo Sr. Don Ramón Rodríguez Arribas, Vicepresidente del Tribunal Constitucional, Magistrados del Tribunal Constitucional, exRectores, autoridades, amigos y compañeros:

Conocí a mi maestro el profesor Torres del Moral hacia el año 1978, cuando la Revista de la Facultad de Derecho de la Universidad Complutense publicó un artículo que yo había escrito como alumno de tercer curso y en el mismo número se publicaba su famoso artículo acerca de Montesquieu. Después coincidí con él en los cursos de doctorado que impartía Don Luis Sánchez Agesta sobre jurisprudencia constitucional.

Aunque hice la tesis en Bolonia y el Relatore de la misma fue De Vergottini, en el año 88 el profesor Torres me invitó a incorporarme a la UNED, lo que acepté inmediatamente con gran entusiasmo y desde entonces ha sido mi maestro. De su mano obtuve la titularidad y la cátedra. Como manifestación de mi agradecimiento, propuse la idea de hacer un libro homenaje, a la que se sumaron con gran empuje los componentes de la comisión organizadora Yolanda Gómez Sánchez, Luis Jimena, Javier Tajadura, Carlos Vidal y Aurora Gutiérrez Nogueroles, y luego todos los participantes en el libro que hoy se presenta.

Antonio ha sido Director del Departamento de Derecho Constitucional de la UNED desde dicho año hasta su jubilación y Director de la Revista Derecho Político de la UNED desde dicho año hasta la actualidad, habiendo dirigido inolvidables números especiales como los dedicados el año 92 a la reforma de la Constitución, los del 25 aniversario de la Constitución, los del homenaje a García Pelayo o los dedicados a la Constitución de Cádiz. Entre artículos, libros 
y capítulos de libros y prólogos, su obra cuenta con más de 200 títulos. No hay ningún ámbito de la asignatura que no haya estudiado y sobre el que no haya publicado. Es el único profesor que conozco con siete sexenios de investigación reconocidos, por más que sólo se puedan devengar económicamente, como es sabido, seis. Ha impartido conferencias y dirigido acciones concertadas con universidades extranjeras: La Sapienza, Bolonia, Bochum, Regensburg, Buenos Aires, Chile, Autónoma de México...

Siendo escaso el tiempo y conociendo la mayoría de los que estamos aquí su obra en el ámbito del Derecho Constitucional, que se extiende a lo largo de los últimos 34 años, se me ha ocurrido que puede tener interés hacer referencia a lo que, utilizando un término de un Proyecto de Investigación que ahora dirige, podríamos llamar su prehistoria, sus años de formación.

Nuestro homenajeado, tras estudiar el bachillerato en Jaén como becario de la Diputación, estudió los dos primeros años de Derecho en la Facultad de Granada los cursos 1959-60 y 1960-61. En otoño de este último año se trasladó a Madrid, donde terminó su licenciatura en Derecho en la Universidad Complutense, simultaneándola con la licenciatura en Filosofía, su otra gran vocación.

Durante los cursos de Granada fue colegial del Colegio Mayor de San Bartolomé y Santiago, el de más tradición en Granada, y conoció a Don Luis Sánchez Agesta, de quien después sería discípulo y con quien trabajaría como ayudante en la Universidad Complutense. De sus estudios de Derecho, fase granadina, recuerda también especialmente a Agustín de Asís Garrote, Catedrático de Filosofía del Derecho, con quien también se reencontraría en Madrid.

Pero fue un estudiante de intereses más amplios que el Derecho, asistiendo a clases de filosofía y arte de la Facultad de filosofía. Lector infatigable, recuerda especialmente de esta época sus lecturas de Unamuno, Machado y Ortega, a lo que se añadía toda la novela contemporánea que caía en sus manos. Con poco tiempo para el descanso, recuerda no obstante sus paseos los fines de semana por la acera del Darro con el periódico debajo del brazo.

En el año 1961, como he dicho, se trasladó a Madrid, conociendo en la Universidad Complutense a las primeras figuras del mundo jurídico de entonces. Entre otros, recuerda a un joven Juan Antonio Carrillo Salcedo, cuando todavía no era ni siquiera numerario, que le impresionó como magnífico profesor. Se reencontró con los profesores Sánchez Agesta y Padilla Serra y también tuvo la ocasión de escuchar imborrables lecciones del profesor Eduardo García de Enterría.

Al mismo tiempo, desde el año 61, cursó la licenciatura de filosofía pura. De las enseñanzas regladas en la Facultad de filosofía le impactó especialmente el estudio de la Gramática histórica de Menéndez Pidal. Pero la personalidad 
más relevante era Aranguren, de cuyo Seminario fue asiduo asistente. Leyó su obra y releyó varias veces su Ética y su Ética y política. En estos años de continua lectura, haciendo de trapero del tiempo, aprovechando incluso los trayectos de metro y autobús, en las ediciones de Austral y Alianza Universidad, forja una base cultural amplia y sólida, leyendo a todos los clásicos. Platón, Aristóteles, Cicerón, Tomás de Aquino, Locke, Montesquieu, Condorcet, cuya importancia resaltaría en su obra posterior, Rousseau, Stuart Mill, Bertrand Russell, los teólogos y juristas españoles de los siglos XVI y XVII, a unos directamente, a otros de modo indirecto, en las obras de Sánchez Agesta, Murillo, Gómez Arboleya, Maravall, etc. También aprendió mucho discutiendo con compañeros, en los que llamaban «encuentros filosóficos». Éste es, como digo; el período en que cimenta una amplia cultura, propia de un humanista.

Al terminar su licenciatura en Derecho, se dirigió a Don Luis Sánchez Agesta para colaborar en su cátedra, quien le contestó que se podía incorporar el sábado inmediatamente posterior. Además, cuando organizó el siguiente curso le asignó un grupo de prácticas.

A partir de este momento, se empapó de la asignatura, de los manuales y monografías, comenzando por la obra de Sánchez Agesta y demás bibliografía que había en el Seminario de Derecho Político de la Facultad.

Todo ello sin perder la vocación humanista. Lee poesía española de los siglos XIX y XX: poesía romántica, Rubén Darío, Manuel Machado, Juan Ramón Jiménez, Generación del 27, Lorca, Alberti, etcétera. Además, desde que vino a Madrid iba una vez al mes al teatro, que le gustaba mucho. Iba a ver todo lo que venía a Madrid y además, leía teatro: Shakespeare, los clásicos franceses, nuestros clásicos, desde La celestina a Benavente, pasando por Lope de Vega. La nostalgia de Andalucía hizo crecer en él la afición al flamenco, en particular al cante jondo. Se hizo con una amplia discografía y asistía a los grandes acontecimientos. Recuerda especialmente a José Meneses, cantaor que cantaba letras compuestas por Moreno Galván.

A pesar de todo esto, terminó su tesis en Derecho: «Ética y poder», sobre la posibilidad de una ética política, en la que vertió todas sus lecturas pasadas, de Platón a Marx.

Un salto cualitativo en su status universitario significó ganar por oposición la adjuntía interina a la cátedra de Sánchez Agesta en el año 72. Dos años después, en el 74, ingresaba por oposición en el cuerpo de Profesores Adjuntos y se convertía así en profesor numerario. En el año 80 ganaría la oposición a Agregado, cuerpo cuyos miembros fueron convertidos en catedráticos por la Ley de Reforma Universitaria. Entre los años 77 y 80 fue Vicedecano de la Facultad de 
Derecho de la Universidad Complutense, siendo Decano Don Mariano Aguilar Navarro y sustituyendo en el Vicedecanato al profesor Gregorio Peces Barba.

En marzo de 1988 leerá su tesis en filosofía, acerca de «La filosofía política durante el Estado moderno: el problema del método». El tema seguirá siendo desarrollado en la actualidad en un proyecto de investigación del que es Investigador principal «La prehistoria del Derecho constitucional», en el que colaboro estudiando las declaraciones de derechos de las colonias inglesas que fundaron Estados Unidos.

Casi ya para terminar, quiero añadir que huelga decir que desde la transición se había volcado en el estudio del Derecho Constitucional. Ya en la primera edición de sus Principios de Derecho constitucional dirá que desea que la Constitución de 1978 sea, con las reformas oportunas, la última de la Nación española. 


\section{INTERVENCIÓN DE ANTONIO TORRES DEL MORAL}

Señor Director del Centro de Estudios Políticos y Constitucionales; Señor Rector Magnífico de la Universidad Nacional de Educación a Distancia, Señor Herrero de Miñón, Consejero Permanente de Estado; autoridades académicas y no académicas presentes; amigos de la Comisión organizadora, compañeros y amigos:

Ante vosotros tenéis lo que va quedando de vuestro amigo Antonio.

¿Qué queréis que os diga? Lo primero, desde luego, es que me siento abrumado por la cantidad y calidad de los asistentes a este acto. Lo segundo, que es difícil sustraerse a la emoción y no terminar diciendo naderías solemnes. Pero haré un esfuerzo.

Quien me conoce bien sabe que, aunque sé degustar de la amistad y agradezco la muy valiosa que me profesáis, no me siento cómodo con sus manifestaciones públicas. Esta ocasión de hoy es diferente. Quizás porque he tenido tiempo de hacerme a la idea, o quizás porque a los setenta años he comenzado a perder un poco de timidez.

\section{CAPÍTULO DE GRACIAS}

Doy vivamente las gracias a la Comisión organizadora, a la que, por economía del acto, personalizo en Yolanda Gómez, que es mi jefa, y en Pedro Julio Tenorio, de quien partió la idea de todo y es, por eso, el máximo culpable.

Gracias, pues, Yolanda, por tus cariñosas palabras. Si de algo estoy tranquilo y seguro es de haber dejado el Departamento en las mejores manos.

Gracias, Pedro. En tu intervención has tenido el buen tino de recordar algunos episodios especialmente significativos de aquellos años que ya no volverán.

Gracias a todos los demás miembros de la Comisión: a Luis Jimena, valenciano de estirpe jiennense y de proyección europea; a Aurora Gutiérrez, gaditana, alma del Departamento; a Carlos Vidal, gallego, que ha llevado el mayor peso en la confección del libro, y a Javier Tajadura, un navarro que explica Derecho Constitucional Español en el País Vasco. 
Muchas gracias, Profesor Álvarez Conde. Si será buena persona Enrique que estrechamos nuestra amistad y la acrecimos desde una oposición a cátedra en la que no lo voté. Gracias, Enrique, por tu amistad y tus muchas muestras de compañerismo.

Gracias igualmente a Miguel Herrero, con cuya amistad me honro. Jurista ilustre, ponente constitucional y doctor honoris causa por mi Universidad, es, además, un universitario vocacional no aprovechado por nuestra Madre Nodriza, en este caso madrastra yerma. Gracias, Miguel, por responder siempre a mis solicitudes de colaboración universitaria.

Gracias a mi Rector, Juan Gimeno, compañero desde hace tanto tiempo y excelente Rector, en quien siempre he encontrado apoyo para mis proyectos y paciencia para con alguna impaciencia mía.

Gracias a Benigno Pendás, Director de este Centro, por su ofrecimiento para el presente acto. Te deseo, Benigno, una eficaz, brillante y feliz gestión de esta Casa.

Esta mi gratitud, larga, ancha y honda, debo hacerla extensiva a todos los compañeros del Departamento, que me han soportado durante mucho tiempo y ahora han querido, con la complicidad del Rector, que siga como profesor emérito.

Hago un recuerdo igualmente amistoso para los compañeros del antiguo Departamento.

A mi Decana saliente, Ana Rosa Martín, y a mi Decana entrante, Mercedes Gómez Adanero, que me honran con su asistencia esta tarde.

Gracias a todos los coautores de este opúsculo, que estoy en la obligación moral de leer entero. Lo haré con mucho gusto, pero no sé cuánto tiempo tardaré en el empeño. Viendo vuestros nombres en el índice (y en algunos textos que ya he leído) no he podido superar dos malditas lágrimas que no me dejaban continuar la lectura. Bueno, os confesaré que yo soy propenso.

Muy especialmente, gracias a quienes habéis querido destacar algo de mi obra o de mi persona: Manuel Ramírez, Juan Carlos López Eismann, Pedro González Trevijano, Miguel Ángel Alegre, Luis Jimena y Pedro Tenorio de nuevo, José M. ${ }^{a}$ Lafuente, Carlos Vidal y Fátima Yáñez, Leyre Burguera y José Ángel Marín. Con toda seguridad os habréis visto obligados a suplir mis carencias sustantivas con adjetivos cariñosos.

Gracias a mi familia: M. ${ }^{a}$ Luisa, Pablo Eugenio, Talia, Gabriel Enzo, mi primer nieto. A mis hermanos Manuel y Juan, y a mis sobrinos, algunos aquí presentes. Muy especialmente, gracias a Isabel y a Eugenio, a quienes tanto debo. 
$\mathrm{Y}$ a todos los dolorosamente ausentes, especialmente a mis padres, que ya hace mucho tiempo me dejaron en la más abatida orfandad.

Mi padre, maestro nacional, fue mi primer y mejor maestro, en una escuela unitaria situada en un sótano de Jaén, en el que de vez en vez tenía que matar alguna rata; él me enseñó todo lo que sé y recuerdo, desde la gramática elemental a las ecuaciones de segundo grado.

Tenía yo diez años y el primer curso de bachillerato recién comenzado cuando por azar escuché una conversación de mi padre con un compañero. Halagaba éste a mi padre diciéndole que yo había salido a él (número uno en sus oposiciones de Magisterio), mientras se quejaba de no poder decir lo mismo de su propio hijo. Mi padre le respondió: «Te equivocas. Mi hijo vale mucho más que yo». No era verdad, pero yo comprendí que tenía el mejor de los padres ${ }^{1}$.

Muchas gracias a mis otros maestros, como Sánchez Agesta. Y a quienes, sin serlo exactamente, me han ayudado mucho, como Alfonso Padilla y Pablo Lucas Verdú, que depositaron su confianza en mí cuando más la precisaba.

Y a mis amigos de Jaén, a los que, pese a la distancia física, he sido siempre fiel, y bien que lo saben.

En fin, muchas gracias a todos los que habéis venido esta tarde a acompañarme; algunos incluso viajando desde lugares lejanos viéndoos obligados a hacer noche en Madrid. Como también al nutrido grupo de compañeros y amigos que no han podido acudir pero han mandado su adhesión a este acto. Os aseguro que no lo olvidaré.

\section{2. ¿UNA DEUDA SALDADA?}

¿Y qué he hecho yo para merecer esto? Pues no lo sé; de verdad, no lo sé. Acaso haber sido fiel a mis amigos y a mí mismo y no dejarme abducir por ningún grupo ni secta aun a costa de pagar un alto precio.

Pedro Tenorio ha contado que estudié el Bachillerato con beca. También fui becario durante la carrera, y después disfruté de una beca de investigación cuyo fruto fue mi primera tesis doctoral. Como igualmente he tenido financiación pública para varios proyectos, uno de los cuales está en vigor. Es decir, no me ha faltado la ayuda de la sociedad española.

Le he correspondido de forma desinteresada y absolutamente gratuita con la dirección y término de 26 tesis doctorales hasta la fecha, cinco de ellas con Pre-

\footnotetext{
Párrafo omitido en el desarrollo del acto.
} 
mio Extraordinario, con la dirección de la Revista de Derecho Político durante 28 años, etcétera. Espero que la deuda esté saldada; si hoy todavía no, sí al final del camino.

Lo mismo digo con la deuda que contraigo con este homenaje. Tendré que acostumbrarme a vivir con estos casi 200 acreedores, además de con los bancos.

\section{TRAYECTORIA PROFESIONAL}

Yo nunca tuve vocación política. No estuve en Paris en mayo del 68 ni guardo un trozo de adoquín, de aquellos que ocultaban la playa de la rive gauche, como tampoco guardo el tique de entrada al concierto de Raimon en la Complutense. Vamos, como político, he sido una calamidad ${ }^{2}$.

En cuanto a mi trayectoria profesional, apenas tengo que aclarar que ha sido muy sencilla.

En los primeros días de estudiante de primer curso de Derecho en Granada, el profesor Agustín de Asís hizo una encuesta entre nosotros, una de cuyas preguntas era qué íbamos a hacer al terminar la carrera. Yo contesté cátedra, lo que motivó alguna sorna suya. Tiempo adelante fuimos amigos, aunque yo nunca me identifiqué como aquel osado estudiante.

Hijo, sobrino y primo de maestros, sólo la enseñanza me llamaba la atención y no me he dedicado a otra cosa. La Universidad ha sido mi mundo y la docencia y la investigación mi vida. No tiene mérito; es que no sé hacer otra cosa.

Gané mi primera oposición por unanimidad; era para Profesor Adjunto Interino; sí, interino por oposición, algo que Juan del Rosal, con el rebuscado lenguaje que solía utilizar, habría calificado como típicamente carpetovetónico. La segunda la empaté porque, aunque obtuve plaza, me dieron una baja puntuación. Y todas las demás las perdí.

Claro que yo podría relatar estas secuencias como aquel general, que describió el retroceso de sus tropas como un avance elástico hacia la retaguardia. Mejor es decirlo con una canción de Jorge Cafrune, que no recuerdo si está en el Martín Fierro:

$$
\begin{aligned}
& \text { «Yo me quitaré el sombrero } \\
& \text { porque así me han enseñao } \\
& \text { y me doy por bien pagao } \\
& \text { entrando tras del primero.» }
\end{aligned}
$$

\footnotetext{
2 Párrafo omitido en el desarrollo del acto.
} 


\section{LA UNED}

Así que bien está lo que bien acaba, porque gané la última con destino directo a la UNED.

Llegué a ella con el por aquel entonces generalizado prejuicio, hoy totalmente superado, de que era una Universidad de segundo orden y con la idea de volver a la Complutense a la primera oportunidad. Dos meses más tarde, y de eso hace 32 años, me juré a mí mismo jubilarme en la UNED, que nació con el valor de la solidaridad como razón de su existencia y está cumpliendo su cometido con alta puntuación.

En ella encontré compañerismo, en buena parte cimentado en las muchas horas que pasamos juntos durante las semanas de exámenes. Encontré un esforzado personal de Administración y Servicios; unos directores de los Centros Asociados entregados a la tarea de hacer del suyo el punto de referencia cultural de la zona; una red de tutores vocacionales y altamente competentes, y unos alumnos que compensan con una enorme motivación su falta de tiempo para el estudio.

Decía García Morente en su ensayo La Universidad: "¿Qué sería de los científicos alemanes sin sus alumnos?» Se refería, claro está, a esos magníficos alumnos que terminaban superándolos. Trasladando la idea a mi Universidad, yo creo que una Matrícula de Honor en la UNED tiene un claro valor añadido y que, en principio, el alumno que la obtiene está facultado para superar a sus maestros, pero casi nunca tiene la oportunidad de demostrarlo.

Yo escribo una carta personal a quienes consiguen tal proeza y después recibo algunas respuestas muy emotivas que guardo como un tesoro.

En cuanto a mis propios discípulos y a quienes tienen la generosidad de considerarse tales, hace tiempo que me superaron, sin que yo me sienta celoso, sino feliz.

La UNED enamora, suele decir su actual Rector. Y es así porque la UNED es un milagro diario.

\section{OBRA ESCRITA}

Hace unos minutos se ha hablado de mi obra escrita. Yo recuerdo haber leído que Hauriou decía que la institución había sido el affaire de su vida. Salvando las distancias, ¿he tenido yo alguno?

Como ha escrito quien hoy nos preside, «el logos debe ir por delante del mito». Por eso, antes de fantasear con ideologías y mitos pujantes, procuré fabricarme 
un logos suficiente con plurales ingredientes, lo cual me ha proporcionado una autoexigencia de precisión y un inmediato rechazo intelectual de los argumentos no concluyentes y del lenguaje jurídico poco meditado. La ciencia no tolera el vale todo ni puede ser construida a ojo de buen cubero.

Mi inclinación primera fue hacia la Filosofía del Derecho, pero finalmente opté por el Derecho Político, aquella disciplina que Nicolás Ramiro Rico describió como adefesio jurídico, hidra de mil cabezas y vertebrado gaseoso. Es gracioso, pero injusto. Algunos cultivadores de aquel Derecho Político nos han dejado obras muy estimables.

De esta primera etapa son mi tesis doctoral —Ética y Poder - y los trabajos sobre Maquiavelo, Hobbes, Montesquieu, Rousseau y Condorcet y sobre Democracia y Representación.

El objeto central de mis estudios de dicha época podía haber sido el binomio democracia-representación, trabajos por los que José María Lafuente me califica en este libro que tengo delante como radical.

Luego, con la transición, y sobre todo con el texto constitucional en vigor, tuve que reciclarme y estudiar Derecho Constitucional; lo tuvimos que hacer muchos, creo que para bien porque en 34 años el Derecho Constitucional español ha alcanzado un nivel notable. Ésta es una explicación, al menos parcial, de la disparidad temática de mis trabajos: hasta 1978 o 1980 y desde entonces para acá.

En esta segunda etapa podría haber hecho una Teoría de la Constitución, o centrarme en el Derecho Electoral y Parlamentario, o en la monarquía, de cuya concepción como monarquía parlamentaria disiente Miguel Herrero sin que eso obste a nuestra amistad; por estos trabajos sobre la monarquía Pedro González Trevijano me llama clásico y algún colega me dijo que me estaba convirtiendo en la reserva espiritual de Occidente; bueno, a éste hace tiempo que lo perdoné.

Recientemente Julia Sevilla decía en un Congreso, después de citarme en su exposición: «¿de qué no ha escrito Torres del Moral?» Lo decía como elogio, pero eso tiene su envés.

El hecho es que no he sido constante en ninguno de esos campos temáticos. Podría decir que sólo he sido constante en la inconstancia. Pero añadiré que no ha habido dispersión, sino inquietud, interés o curiosidad por los más variados problemas jurídico-políticos, además de atención prioritaria a urgencias propias y de compañeros. Con deciros que no tengo publicada mi segunda tesis doctoral es suficiente. De eso hace 25 años. Nunca he encontrado tres meses para acondicionarla y quedar satisfecho de ella. Espero hacerlo pronto, aunque me cueste.

Por otra parte, nunca he abandonado aquellas primeras inclinaciones, como tampoco la reflexión sobre la política y su dimensión ética, tantas veces olvidada en la práctica y teorizada con vaciedades. 
En resumen, todo ha sido un ir y venir estudiando, colmando mis lagunas e intentando poner en orden mis ideas para poder enseñar algo coherente a los alumnos; o, por decirlo de nuevo con palabras de García Morente, para transmitir no un saber diletante, sino "un saber pensado».

Debo confesar, por tanto, que esta tortuga del Derecho Constitucional ha resultado ser demasiado rápida para un Aquiles tan torpe como yo. Así llego a este final de etapa ayuno de trofeos. Pero seguiré en el empeño hasta el término de la carrera, aunque ya adivino el resultado. De manera que me iré habiéndolo intentado, pero con la relativa insatisfacción del deber no del todo bien cumplido.

\section{6. ÚLTIMA ETAPA}

Sin embargo, nunca he caído en la melancolía, porque siempre he sabido que había que seguir y aprovechar el tiempo. Éste acaso sea mi único rasgo positivo: aprovecho mucho el tiempo porque me concentro con facilidad. Apenas hay tiempos muertos, salvo para una buena tertulia, que en realidad es tiempo vivo y muy vivo.

Si Shakespeare le hizo decir a Ricardo III «Un caballo, mi reino por un caballo», yo me he dicho a mí mismo muchas veces: «mi reino por una conversación inteligente». No hay placer comparable, salvo un rato de silencio vivido con intensidad.

Pues bien, respecto de esta rara facilidad mía, Joan Oliver suele contar con evidente hipérbole que yo escribí un artículo para la Revista de Estudios Políticos en las servilletas de una cafetería. No fue así exactamente; todo el artículo, no.

Fue hace treinta y tantos veranos. Estaba yo atrancado con mi artículo sobre Rousseau dando vueltas (más que paseando) por la Alameda de Marbella. Cuando logré desbrozar el nudo argumental que se me resistía, me senté en la terraza de una cafetería, emborroné ocho o diez servilletas y me fui rápidamente a casa para incorporarlas al artículo. Ese día me quedé sin playa.

Últimamente, mis discípulos y amigos están convirtiéndome en prologuista. El prólogo es un género que cultivo con gusto. Ya he escrito 47, el último a un magnífico libro de Luis Jimena sobre Jurisdicción nacional y control de convencionalidad, aunque aún no se lo he entregado.

No pretendo compararlos con el que Xavier Zubiri escribió en 1940 para la Historia de la Filosofía de un joven filósofo llamado Julián Marías, pero tampoco son prólogos de cuartilla y media ensalzando la inteligencia del autor, sino amplios y terciando en el objeto del libro. 
En los últimos he proyectado alguna pretensión literaria; verbigracia, en el que he escrito para el excelente libro de Pedro Tenorio sobre Constitución, derechos fundamentales y seguridad ${ }^{3}$.

En fin, acabaré confesando lo que ya se ha dicho: a mí lo que me gusta de verdad es el teatro, el flamenco y la poesía.

Y así, en los últimos años, dedico los viajes en avión y en tren a escribir chifladuras, desvaríos, en vez de ver películas infames.

Después he mandado esas cuartillas a mis compañeros de Departamento y a algunos amigos. Y, como son muy educados y no protestan, los he torturado sádicamente con puntuales entregas en Navidad y fin de curso.

Tengo además un crítico literario excelente e implacable: Carlos Vidal padre, magnífico abogado de El Ferrol y mejor poeta, que me hace dos tipos de críticas:

a) Las favorables, que son las menos, me las envía por escrito.

b) Y las desfavorables las deduzco yo de su silencio.

Una muestra de estas agresiones a las buenas letras la tenéis a la salida. Se trata de un pequeño homenaje a Antonio Machado, o, como diría Borges, al hermano de Manuel. Ya estáis viendo qué poco original soy:

- Como persona, no tengo más patrimonio que vuestra amistad.

- Como aprendiz malogrado de juntaletras sin pretensiones, soy un andaluz que ama Castilla.

Por eso, termino con unos versos sencillos de esa muestra:

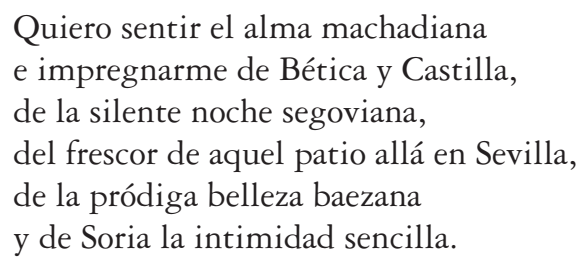

Y ya no puedo decir nada más. De verdad, no puedo. Sólo deseo que me sigáis queriendo como yo os quiero.

Gracias y hasta siempre.

3 Párrafo omitido en el desarrollo del acto. 